\title{
Subdiffusive and superdiffusive quantum transport and generalized duality
}

\author{
Maura Sassetti \\ Istituto di Fisica di Ingegneria, Istituto Nazionale di Fisica della Materia, Università di Genova, I-16146 Genova, Italy \\ Henning Schomerus \\ Fachbereich Physik, Universität-Gesamthochschule Essen, D-45117 Essen, Germany \\ Ulrich Weiss \\ Institut für Theoretische Physik, Universität Stuttgart, D-70550 Stuttgart, Germany
}

(Received 16 November 1995)

\begin{abstract}
As a generic model for transport of interacting fermions through a barrier or interstitials in a lattice, quantum Brownian motion in a periodic potential is studied. There is a duality transformation between the continuous coordinate or phase representation and the discrete momentum or charge representation for general frequencydependent damping. Sub-Ohmic friction is mapped on super-Ohmic friction, and vice versa. The mapping is exact for arbitrary barrier height and valid at any temperature. Thus all features of the continuous model can be investigated from analytical or numerical analysis of the discrete model. Nonperturbative results for the frequency-dependent linear mobility including subdiffusive and superdiffusive behaviors are reported.
\end{abstract}

Quantum Brownian motion in a periodic potential (QBM) is a generic model for many transport phenomena in condensed matter. ${ }^{1}$ Major themes in the past have been the phenomena of quantum coherence and localization. Transport of charge in one-dimensional interacting Fermi systems through barriers or impurities within the continuous $\theta$-phase representation of the Luttinger-liquid model ${ }^{2}$ is also described by the above model. The harmonic liquid in the fermionic model away from the barrier corresponds to the thermal reservoir in the QBM model, and the conductance in the first model corresponds to the mobility in the latter. Short-range electron-electron interaction with coupling parameter $g$ $(g<1$ in the repulsive case) corresponds to Ohmic damping in the connected QBM model, and $g$ is related to the Ohmic damping parameter $\alpha$ by $g=1 / \alpha$. Moreover, unscreened long-range Coulomb repulsion ${ }^{3}$ corresponds to sub-Ohmic damping in the related QBM model, ${ }^{4}$ leading to strong suppression of the conductance at low temperatures. A variety of other physical or chemical systems involves transport of charge through barriers for Ohmic or super-Ohmic damping. 5,6

Schmid has shown for Ohmic damping ${ }^{7}$ that the continuous QBM transport problem at $T=0$ can be mapped on a tight-binding model. The mapping resulted in a duality transformation for the dc mobility in which diffusive and localized behavior are interchanged. The mapping was generalized later to finite $T{ }^{1}$ Similarly, the continuous phase representation of the fermionic problem is directly related to a discrete charge representation.

In this paper, we generalize the mapping to frequencydependent damping, e.g., unscreened Coulomb interactions in the fermionic problem. ${ }^{3,4}$ Our study will reveal an exact mapping between the respective Hamiltonians. Then, upon using the equations of motion, an exact duality relation between the respective frequency-dependent linear mobilities or conductances will be derived. Results exhibiting superdiffusive, diffusive, subdiffusive, and strictly localized behaviors, depending on the parameter regime, will be given.
Here we restrict the attention to the QBM transport problem, as the transcription into the fermionic problem is straightforward. ${ }^{2}$

As another essential reason for our study, it should be stressed that it is important to perform numerical real-time simulations of the dynamics within the equivalent discrete model. The discrete variables significantly reduce the relevant configuration space subject to Monte Carlo sampling compared to the continuous model, and meaningful real-time simulations in the interesting nonperturbative lowtemperature regime are possible. ${ }^{9}$

The system-plus-bath Hamiltonian for a Brownian particle of mass $M$ moving in a washboard potential (WB) with period $Q_{0}$ and corrugation strength $V_{0}$ is

$$
\begin{aligned}
H_{\mathrm{WB}}= & P^{2} / 2 M-V_{0} \cos \left(2 \pi Q / Q_{0}\right) \\
& +\sum_{\alpha}\left[\frac{p_{\alpha}^{2}}{2 m_{\alpha}}+\frac{m_{\alpha} \omega_{\alpha}^{2}}{2}\left(x_{\alpha}-\frac{c_{\alpha}}{m_{\alpha} \omega_{\alpha}^{2}} Q\right)^{2}\right] .
\end{aligned}
$$

Here we have included the usual potential renormalization term $\Sigma_{\alpha}\left(c_{\alpha}^{2} / 2 m_{\alpha} \omega_{\alpha}^{2}\right) Q^{2}$ which provides invariance of the coupling $\Sigma_{\alpha} c_{\alpha} x_{\alpha} Q$ under spatial translations. As far as we are interested in properties of the particle, the coupling constants $c_{\alpha}$ and the parameters of the bath are relevant only via the spectral density of the coupling $J_{\mathrm{WB}}(\omega)$ $=(\pi / 2) \Sigma_{\alpha}\left(c_{\alpha}^{2} / m_{\alpha} \omega_{\alpha}\right) \delta\left(\omega-\omega_{\alpha}\right)$.

The dual tight-binding (TB) model with transfer matrix element $V_{0}$ and translational-invariant coupling is

$$
\begin{aligned}
H_{\mathrm{TB}}= & -\frac{V_{0}}{2}\left(e^{i q_{0} P / \hbar}+\text { H.c. }\right) \\
& +\sum_{\alpha}\left[\frac{\pi_{\alpha}^{2}}{2 M_{\alpha}}+\frac{M_{\alpha} \Omega_{\alpha}^{2}}{2}\left(u_{\alpha}-\frac{d_{\alpha}}{M_{\alpha} \Omega_{\alpha}^{2}} Q\right)^{2}\right], \\
Q & =q_{0} \sum_{n} n a_{n}^{\dagger} a_{n} ; \quad e^{i q_{0} P / \hbar}=\sum_{n} a_{n}^{\dagger} a_{n+1},
\end{aligned}
$$

where $q_{0}$ is the TB lattice constant. In the discrete representation, the operators $a_{n}^{\dagger}$ and $a_{n}$ create and annihilate a par- 
ticle at the site $n$, respectively. The spectral density of the environmental coupling in the tight-binding model is $J_{\mathrm{TB}}(\omega)=(\pi / 2) \Sigma_{\alpha}\left(d_{\alpha}^{2} / M_{\alpha} \Omega_{\alpha}\right) \delta\left(\omega-\Omega_{\alpha}\right)$.

In the first step of the mapping of (1) onto (2), we perform the canonical transformations $\left(\kappa=2 \pi \hbar / Q_{0} q_{0}\right)$,

$$
\begin{gathered}
p_{\alpha} \rightarrow-m_{\alpha} \omega_{\alpha} x_{\alpha}, \quad x_{\alpha} \rightarrow p_{\alpha} / m_{\alpha} \omega_{\alpha}+P c_{\alpha} / \kappa m_{\alpha} \omega_{\alpha}^{2}, \\
Q \rightarrow P / \kappa, \quad P \rightarrow-\kappa Q+\sum_{\alpha} c_{\alpha} x_{\alpha} / \omega_{\alpha} .
\end{gathered}
$$

In the intermediate form of the Hamiltonian, the modes of the environment are coupled with each other. The corresponding term of the Hamiltonian is

$$
H_{\mathrm{R}}=\sum_{\alpha}\left[\frac{p_{\alpha}^{2}}{2 m_{\alpha}}+\frac{m_{\alpha} \omega_{\alpha}^{2} x_{\alpha}^{2}}{2}\right]+\frac{1}{2 M}\left(\sum_{\alpha} \frac{c_{\alpha} x_{\alpha}}{\omega_{\alpha}}\right)^{2} .
$$

In the next step, we diagonalize $H_{R}$, thereby introducing canonical variables $\pi_{\alpha}$ and $u_{\alpha}$, and parameters $M_{\alpha}$ and $\Omega_{\alpha}$. By the transformation, the interaction term $(\kappa Q / M) \Sigma_{\alpha} c_{\alpha} x_{\alpha} / \omega_{\alpha}$ is changed into $Q \Sigma_{\alpha} d_{\alpha} u_{\alpha}$.

The mapping of (1) onto (2) is completed on imposing spatial invariance of the coupling $\Sigma_{\alpha} d_{\alpha} u_{\alpha} Q$,

$$
\kappa^{2} / M=\sum_{\alpha} d_{\alpha}^{2} / M_{\alpha} \Omega_{\alpha}^{2}
$$

By (3), the mapping is constrained, as we shall see.

The relation between the spectral functions $J_{\mathrm{WB}}(\omega)$ and $J_{\mathrm{TB}}(\omega)$ results from properties of the dynamical matrix $A_{\alpha \beta}(\omega)$ of the reservoir Hamiltonian $H_{R}$. We have $A_{\alpha \beta}(\omega)=B_{\alpha \beta}(\omega)+c_{\alpha} c_{\beta} / M \omega_{\alpha} \omega_{\beta}$, where $B_{\alpha \beta}(\omega)$ $=\delta_{\alpha \beta} m_{\alpha}\left(\omega_{\alpha}^{2}-\omega^{2}\right)$. Observing that the coupling terms are in the form of an exterior vector product, it is straightforward to calculate the ratio of the determinants of the matrices $A$ and $B, \Delta^{-1}(\omega) \equiv \operatorname{det} A(\omega) / \operatorname{det} B(\omega)$,

$$
\begin{aligned}
\Delta^{-1}(\omega) & =1+\frac{1}{M} \sum_{\alpha} \frac{c_{\alpha}^{2}}{m_{\alpha} \omega_{\alpha}^{2}\left(\omega_{\alpha}^{2}-\omega^{2}\right)} \\
& =1+i \gamma_{\mathrm{WB}}(\omega) / \omega .
\end{aligned}
$$

In the second line, we have introduced the spectral damping function $\gamma_{\mathrm{WB}}(\omega)$. The function $\gamma(\omega)$ is defined in terms of the continuous spectral density of the coupling $J(\omega)$ of the respective model by

$$
\gamma(\omega)=\lim _{\epsilon \rightarrow 0^{+}} \frac{2 \omega}{i \pi M} \int_{0}^{\infty} d \omega^{\prime} \frac{J\left(\omega^{\prime}\right)}{\omega^{\prime}\left(\omega^{\prime 2}-\omega^{2}-i \epsilon \operatorname{sgn} \omega\right)} .
$$

On the other hand, we may resolve $A_{\alpha \beta}$ for $B_{\alpha \beta}$ and then switch to the unitarily equivalent form in which $A$ is diagonal, $\tilde{A}_{\alpha \beta}(\omega)=\delta_{\alpha \beta} M_{\alpha}\left(\Omega_{\alpha}^{2}-\omega^{2}\right)$. We then have $\tilde{B}_{\alpha \beta}(\omega)$ $=\tilde{A}_{\alpha \beta}(\omega)-\left(M / \kappa^{2}\right) d_{\alpha} d_{\beta}$. From this we find

$$
\begin{aligned}
\Delta(\omega) & =1-\frac{M}{\kappa^{2}} \sum_{\alpha} \frac{d_{\alpha}^{2}}{M_{\alpha}\left(\Omega_{\alpha}^{2}-\omega^{2}\right)} \\
& =-i\left(M^{2} / \kappa^{2}\right) \omega \gamma_{\mathrm{TB}}(\omega),
\end{aligned}
$$

where in the second line we have used (3), and where $\gamma_{\mathrm{TB}}(\omega)$ is the damping function of the TB model. Combin- ing (4) with (6), we obtain an exact relation between the damping functions of the two models,

$$
\gamma_{\mathrm{TB}}(\omega)\left[\gamma_{\mathrm{WB}}(\omega)-i \omega\right]=\kappa^{2} / M^{2}
$$

With the relation $J(\omega)=M \omega \operatorname{Re} \gamma(\omega)$, we then have

$$
\begin{gathered}
J_{\mathrm{WB}}(\omega)=\left(\kappa^{2} / M^{2}\right) J_{\mathrm{TB}}(\omega) /\left|\gamma_{\mathrm{TB}}(\omega)\right|^{2}, \\
J_{\mathrm{TB}}(\omega)=\left(\kappa^{2} / M^{2}\right) J_{\mathrm{WB}}(\omega) /\left|\omega+i \gamma_{\mathrm{WB}}(\omega)\right|^{2} .
\end{gathered}
$$

Thus, by use of (5), the spectral density of the one model can be calculated for any form of the spectral density of the other model.

To be definite, let us assume a simple power-law form for the spectral density of the WB model in the entire relevant frequency range,

$$
J_{\mathrm{WB}}(\omega)=\eta_{s} \tilde{\omega}(\omega / \tilde{\omega})^{s}, \quad 0<s<2,
$$

resulting in the spectral damping function,

$$
\gamma_{\mathrm{WB}}(\omega)=\gamma_{s}(-i \omega / \tilde{\omega})^{s-1},
$$

with $\gamma_{s}=\eta_{s} /[M \sin (\pi s / 2)]$. Here we have introduced for $s \neq 1$ a reference frequency $\tilde{\omega}$ so that the coupling strength $\gamma_{s}$ has the usual dimension of frequency. The case $s=1$ describes (frequency-independent) Ohmic dissipation, while the cases $s<1$ and $s>1$ are usually referred to as sub-Ohmic and super-Ohmic friction.

We are now in the position to make a check on the consistency of the condition (3). There follows from (6) that the constraint (3) is satisfied if $\Delta(\omega)$ vanishes at zero frequency. Now, upon inserting (11) in (4), we see that the condition $\lim _{\omega \rightarrow 0} \Delta(\omega) \rightarrow 0$ is satisfied for $s$ in the range $0<s<2$. For $s \geqslant 2$, we have $\gamma_{\mathrm{WB}}(\omega \rightarrow 0) \propto \omega$. Damping becomes ineffective in this limit, and the only remaining effect is mass renormalization. ${ }^{10}$

We have thus demonstrated an exact duality between the weak corrugation model (1) and the tight-binding model (2). In the mapping, the continuous coordinate $Q$ in the WB model is identified, up to a scale factor $1 / \kappa$, with the quasimomentum $P$ in the dual TB model. The mapping is possible only for nonvanishing dissipation since otherwise the scale factor is infinity. Strictly speaking, the mapping holds under the condition $\lim _{\omega \rightarrow 0} \omega^{2} / J(\omega) \rightarrow 0$. There are no restrictions on the form of $J(\omega)$ at finite frequencies except those enforced on physical grounds. There follows from (8) or (9) that the spectral density $J_{\mathrm{WB}}(\omega) \propto \omega^{s}$ of the weak corrugation model maps on the spectral density $J_{\mathrm{TB}}(\omega \rightarrow 0) \propto \omega^{s^{\prime}}$ of the dual TB model, where $s^{\prime}=2-s$. Thus formally in the spectral densities of the coupling, the power $s$ is mapped on the power $2-s$. Physically, this means that sub-Ohmic and super-Ohmic friction are interchanged in the transformation, while Ohmic friction is mapped on Ohmic friction.

Upon inserting (10) into (5) and using (9), the spectral density of the dual TB model takes the form

$$
J_{\mathrm{TB}}(\omega)=\frac{\kappa^{2}}{\eta_{s}} \frac{\tilde{\omega}(\omega / \tilde{\omega})^{2-s}}{1+\left[\cot (\pi s / 2)-\left(M \tilde{\omega} / \eta_{s}\right)(\omega / \tilde{\omega})^{2-s}\right]^{2}} .
$$

In contrast to the chosen form for $J_{\mathrm{WB}}(\omega)$, the function $J_{\mathrm{TB}}(\omega)$ exhibits a soft cutoff. Physically, this is due to the finite mass of the particle in the washboard potential model 
which provides a natural cutoff in the spectral density. In the Ohmic case, the expression for $J_{\mathrm{TB}}(\omega)$ reduces to the Drude form, ${ }^{1} J_{\mathrm{TB}}(\omega) \propto \omega /\left[1+\left(\omega / \gamma_{1}\right)^{2}\right]$.

After having completed the mapping of the Hamiltonians, we turn to a study of the respective linear ac mobilities. First, we observe that by the above unitary transformations a coordinate autocorrelation function of the WB model is transformed into a momentum autocorrelation function of the associated TB model. From this we find for the linear mobility of the WB model

$$
\mu_{\mathrm{WB}}(\omega)=-i \omega X_{\mathrm{TB}}(\omega) / \kappa^{2},
$$

where $X_{\mathrm{TB}}(\omega)$ is the Fourier transform of the retarded momentum response function of the TB model. On the other hand, the ac mobility of the TB model is related to the Fourier transform $Y_{\mathrm{TB}}(\omega)$ of the respective retarded coordinate response function of the TB model by

$$
\mu_{\mathrm{TB}}(\omega)=-i \omega Y_{\mathrm{TB}}(\omega) .
$$

To relate $X_{\mathrm{TB}}(\omega)$ to $Y_{\mathrm{TB}}(\omega)$, we use the equations of motion resulting from (2). This yields

$$
\omega^{2} X_{\mathrm{TB}}(\omega)=i M \omega \gamma_{\mathrm{TB}}(\omega)-M^{2} \omega^{2} \gamma_{\mathrm{TB}}^{2}(\omega) Y_{\mathrm{TB}}(\omega) \text {. }
$$

Using this, and the relations (12), (13), and (7), we find for the linear ac mobility the exact relations

$$
\begin{aligned}
\mu_{\mathrm{WB}}(\omega)= & \frac{M}{\kappa^{2}} \gamma_{\mathrm{TB}}(\omega)-\frac{M^{2}}{\kappa^{2}} \gamma_{\mathrm{TB}}^{2}(\omega) \mu_{\mathrm{TB}}(\omega), \\
\mu_{\mathrm{TB}}(\omega)= & \left(M / \kappa^{2}\right)\left[\gamma_{\mathrm{WB}}(\omega)-i \omega\right] \\
& -\left(M^{2} / \kappa^{2}\right)\left[\gamma_{\mathrm{WB}}(\omega)-i \omega\right]^{2} \mu_{\mathrm{WB}}(\omega) .
\end{aligned}
$$

The expressions (7)-(9), (14), and (15) are the central relations of the duality. Since they have been derived by using canonical transformations and commutation relations, they are not restricted to the discrete representation in (2). Most remarkably, the duality holds for any spectral form of the damping kernel, with the only restriction that damping remains effective at zero frequency. Thus, there is a far more general duality between these models than considered in earlier studies. ${ }^{1,7,8}$ Here, the correspondence is shown for the frequency-dependent linear mobility. It is important that no restrictions were placed on $T$ and on $V_{0}$.

So far, we have considered the length scales $Q_{0}$ and $q_{0}$ as free parameters. In order to simplify the relation between the ac mobilities, we now make the specific choice

$$
M \gamma_{s} Q_{0} q_{0} / 2 \pi \hbar \equiv M \gamma_{s} / \kappa=1 .
$$

Then the standard dimensionless coupling parameters $\alpha_{\mathrm{WB}}=: M \gamma_{s} Q_{0}^{2} / 2 \pi \hbar$ and $\alpha_{\mathrm{TB}}=: M \gamma_{s} q_{0}^{2} / 2 \pi \hbar$ are related by $Q_{0} / q_{0}=\alpha_{\mathrm{WB}}=1 / \alpha_{\mathrm{TB}}$.

In the remainder of the discussion, we confine ourselves to the low-frequency regime $\omega / \gamma_{\mathrm{WB}}(\omega) \ll 1$, in which the relations (14) and (15) become symmetric. Upon using (7), (11), and (16), we obtain the relation $\left(\mu_{0}=: 1 / M \gamma_{s}\right)$

$$
\mu_{\mathrm{WB}}(\omega)=(i \tilde{\omega} / \omega)^{s-1} \mu_{0}-(i \tilde{\omega} / \omega)^{2 s-2} \mu_{\mathrm{TB}}(\omega) .
$$

For $s=1$, this reduces to the duality relation of the Ohmic case, $\mu_{\mathrm{TB}}(\omega)+\mu_{\mathrm{WB}}(\omega)=\mu_{0}$. Most analytic work on Ohmic friction has been restricted to the TB model with a very high cutoff frequency of the bath modes. Much of the theoretical interest has been concentrated on the localization transition at $T=0$ at friction strength $\alpha_{\mathrm{TB}}=1$ and on the renormalization group flows. For a discussion of this, and of the implications of the duality relation for the WB model, we refer to the literature. ${ }^{1,2,11,12}$ In many interesting cases, e.g., charge transfer in chemical reactions, the density $J_{\mathrm{WB}}(\omega)$ is not in the simple power-law form (10), and also the finite bandwidth $\omega_{c}$ may have important influence on the dynamics. Nevertheless, the associated spectral density $J_{\mathrm{TB}}(\omega)$ is given by (9), and one may now take advantage of performing numerical simulations of the equivalent TB model. We wish to emphasize that even the crossover from quantum tunneling to thermal hopping across the barrier can be studied in the equivalent TB model.

According to the generalization given here, important conclusions can now be drawn also for non-Ohmic damping. In the sequel, we present results obtained by analytic methods for the TB model at low temperatures and highfrequency cutoff. Upon using (17), we then describe the implications for the dual WB model.

Consider now first the TB model for super-Ohmic damping. It has been shown in earlier work ${ }^{5,13}$ that the TB model for $1<s^{\prime}<2$ is perturbative in $V_{0}^{2}$ at finite $T$ and has a diffusive limit $\mu_{\mathrm{TB}}(\omega \rightarrow 0)=\bar{\mu}$, where $\bar{\mu}$ depends on temperature and the leading term is of order $V_{0}^{2}$. With decreasing temperature, the effective transfer matrix element of the super-Ohmic TB model scales to higher values for fixed $\omega$, and for $T=0$ and $\omega \rightarrow 0$ it even scales to infinity. Hence the model becomes nonperturbative in $V_{0}^{2}$ in this limit. We have been able to perform the resummation of the full power series in $V_{0}^{2} \cdot{ }^{14}$ Interestingly, we find that the asymptotic behavior is independent of $V_{0}$ and is $\mu_{\mathrm{TB}}(\omega \rightarrow 0)$ $=(-i \omega / \tilde{\omega})^{1-s^{\prime}} \mu_{0}$. Thus, the tight-binding lattice is completely dissolved in this limit, and the mobility is that of a free super-Ohmic Brownian particle. In linear response, the mean position grows superdiffusively, ${ }^{10}\langle Q(t)\rangle \propto t^{s^{\prime}}$.

In contrast, the sub-Ohmic TB model $\left(s^{\prime}<1\right)$ is nonperturbative in $V_{0}^{2}$ at finite $T$ in the limit $\omega \rightarrow 0$. We have been able again to sum the full power series in $V_{0}^{2},{ }^{13,14}$ yielding subdiffusive behavior $\mu_{\mathrm{TB}}(\omega \rightarrow 0)=(-i \omega / \tilde{\omega})^{1-s^{\prime}} \mu_{0}$, and the subleading term is $\propto\left(1 / V_{0}^{2}\right)(-i \omega / \tilde{\omega})^{2-2 s^{\prime}}$. The linear mobility vanishes in the dc limit. Regarding the behavior in the time regime, in linear response, the mean position $\langle Q(t)\rangle$ grows sluggishly with subdiffusive power law $t^{s^{\prime}}$. As the temperature is decreased, the effective transfer matrix element of the sub-Ohmic TB model scales to lower values for fixed $\omega$, and at $T=0$ it vanishes with an essential singularity $\propto \exp (-\mathrm{const} / \omega)$ as $\omega \rightarrow 0$. Hence the mobility vanishes faster than any power of $\omega$ in the zero-frequency limit, and the particle gets strictly localized.

Upon using these results of the TB model and the relation (17) with the mapping $s^{\prime} \rightarrow 2-s$, we now immediately get results for the mobility of the WB model.

The linear mobility of the sub-Ohmic WB model at finite $T$ is $\mu_{\mathrm{WB}}(\omega \rightarrow 0)=(-i \omega / \tilde{\omega})^{1-s} \mu_{0}$, and the subleading correction is $-(-i \omega / \tilde{\omega})^{2-2 s} \bar{\mu}$. The potential represents an irrelevant perturbation in this regime. The mean position of the 
particle $\langle Q(t)\rangle$ grows subdiffusively $\propto t^{s}$ with $s<1,{ }^{10}$ i.e., just as in the absence of the potential, and the leading potential corrections grow as $t^{2 s-1}$, which is in agreement with the findings in Ref. 15. At $T=0$, the term $\propto \omega^{1-s}$, representing the free Brownian particle, is canceled by the asymptotic expression resulting from the full power series in $V_{0}^{2}$. As a result, the mean position of the particle grows even slower than in the free sub-Ohmic case.

In the super-Ohmic WB model at finite $T$, the superdiffusive contribution of the free Brownian motion is canceled once again, and we find slower diffusive behavior, $\mu_{\mathrm{WB}}(\omega \rightarrow 0)=$ const. On the contrary, at $T=0$ the potential is an irrelevant perturbation in the super-Ohmic WB model. Thus we obtain the superdiffusive characteristics of the free Brownian particle $\mu_{\mathrm{WB}}(\omega \rightarrow 0)=(-i \omega / \tilde{\omega})^{1-s} \mu_{0}$ in this limit.

Considering the fact that the system becomes increasingly sensitive to its low-frequency properties as the temperature is decreased, the above characteristics are fully consistent with physical intuition. For sub-Ohmic damping and decreasing temperature, the mobility is progressively suppressed at constant low frequency in both models. This is due to the diverging spectral damping function $\gamma(\omega)$ for $\omega \rightarrow 0$. On the contrary, for super-Ohmic damping the mobility is enhanced with decreasing temperature in both models since the respective spectral damping function vanishes at zero frequency.

The duality relations presented here are directly relevant to numerical simulation of quantum transport in a continuous periodic potential for any form of the spectral damping func- tion. Using the "bosonized" language, charge transfer in a Luttinger liquid through a barrier or impurity ${ }^{2}$ is also described by models of the form (1) or (2). Hence, the results also apply to charge transport in correlated fermion systems. For instance, the linear conductance $G(\omega)$ of the impurity scattering problem is related to the linear mobility $\mu(\omega)$ in the washboard potential with period $Q_{0}$ by $G(\omega)$ $=e^{2} \mu(\omega) / Q_{0}^{2}$. Further, the equilibrium noise spectrum of the current is given by $S(\omega)=\hbar \omega \operatorname{coth}\left(\hbar \omega / 2 k_{\mathrm{B}} T\right) G(\omega)$. The interesting case of unscreened Coulomb interactions in the impurity scattering problem, ${ }^{4}$ and in tunneling between edge states of fractional quantum Hall liquids, ${ }^{16}$ corresponds to logarithmically diverging sub-Ohmic behavior, $\gamma(\omega \rightarrow 0)$ $\propto-\ln (\omega / \tilde{\omega})$.

In conclusion, we have shown that there exists a general duality transformation between a quantum Brownian particle in a continuous washboard potential and a dissipative tightbinding model. Because the tight-binding approximation ignores excited states at each lattice site, one might think that a general mapping between the two models is impossible. We have shown, however, that the tight-binding model is fully sensitive to all aspects of both the quantum and the thermal hopping dynamics of the continuous model. In fact, the tightbinding model is reconciled with the continuous model by properly taking into account the change in the spectral density.

We thank R. Egger and L.S. Schulman for valuable discussions.
${ }^{1}$ M.P.A. Fisher and W. Zwerger, Phys. Rev. B 32, 6190 (1985).

${ }^{2}$ C.L. Kane and M.P.A. Fisher, Phys. Rev. Lett. 68, 1220 (1992); Phys. Rev. B 46, 15233 (1992).

${ }^{3}$ H.J. Schulz, Phys. Rev. Lett. 71, 1864 (1992).

${ }^{4}$ M. Fabrizio, A.O. Gogolin, and S. Scheidl, Phys. Rev. Lett. 72, 2235 (1994).

${ }^{5}$ A.J. Leggett, S. Chakravarty, A.T. Dorsey, M.P.A. Fisher, and W. Zwerger, Rev. Mod. Phys. 59, 1 (1987).

${ }^{6}$ Y. Kagan, J. Low Temp. Phys. 87, 525 (1992).

${ }^{7}$ A. Schmid, Phys. Rev. Lett. 51, 1506 (1983).

${ }^{8}$ G. Schön and A.D. Zaikin, Phys. Rep. 198, 237 (1990).
${ }^{9}$ K. Leung, R. Egger, and C.H. Mak, Phys. Rev. Lett. 75, 3344 (1995).

${ }^{10}$ H. Grabert, P. Schramm, and G.-L. Ingold, Phys. Rep. 168, 115 (1988).

${ }^{11}$ F. Guinea, V. Hakim, and A. Muramatsu, Phys. Rev. Lett. 54, 263 (1985).

${ }^{12}$ W. Zwerger, Phys. Rev. B 35, 4737 (1987).

${ }^{13}$ U. Weiss, M. Sassetti, T. Negele, and M. Wollensak, Z. Phys. B 84, 471 (1991).

${ }^{14}$ M. Sassetti, H. Schomerus, R. Egger, and U. Weiss (unpublished).

${ }^{15}$ Y.-C. Chen and J.L. Lebowitz, Phys. Rev. B 46, 10743 (1992).

${ }^{16}$ X.G. Wen, Phys. Rev. B 44, 5708 (1991). 